\title{
Un modelo estructural del consumo de alcohol entre estudiantes de preparatoria
}

\section{A structural model of alcohol consumption among high school students}

\author{
Javier Aguilar Villalobos \\ Amira Aguilar Casis \\ Carolina Sarmiento Silva \\ Alejandra Valencia Cruz \\ Universidad Nacional Autónoma de México
}

\begin{abstract}
Resumen
El objetivo del presente estudio fue desarrollar y probar un modelo estructural del consumo de bebidas alcohólicas entre estudiantes de bachillerato, con base en los resultados de una investigación similar realizada con estudiantes de licenciatura, además de efectuar un análisis diferencial de resultados entre ambos sondeos. La muestra estaba conformada por 190 estudiantes de preparatoria, a quienes se aplicaron las siguientes escalas: motivos para beber (conformidad, afrontamiento y mejoramiento-social), expectativas negativas, problemas, amigos bebedores, asistencia a fiestas, búsqueda de sensaciones y tres medidas de consumo, elaboradas y adaptadas en el estudio de licenciatura. Posteriormente, se validaron las escalas mediante análisis de regresión múltiple y se elaboró un modelo estructural del consumo de bebidas alcohólicas, el cual mostró un ajuste adecuado a los datos de acuerdo con los índices más aceptados. El modelo obtenido mostró que el consumo de alcohol tuvo relación directa con los motivos mencionados, las expectativas negativas y la asistencia a fiestas, e indirectamente por la búsqueda de sensaciones y por amigos bebedores. Las diferencias más notables entre los estudiantes de bachillerato y de licenciatura son la mayor resistencia al consumo por presión de grupo en los bachilleres y la mayor consciencia de los riesgos de consumo entre los universitarios.

Palabras clave: modelo estructural, consumo de alcohol, estudiantes de preparatoria, motivos para beber, problemas.
\end{abstract}

Nota del autor

Javier Aguilar Villalobos, Facultad de Psicología, Universidad Nacional Autónoma de México; Amira Aguilar Casis, Facultad de Psicología, Universidad Nacional Autónoma de México; Carolina Sarmiento Silva, Escuela Nacional Preparatoria Plantel No. 8, Universidad Nacional Autónoma de México; Alejandra Valencia Cruz, Facultad de Psicología, Universidad Nacional Autónoma de México. Esta investigación fue subvencionada por el Programa de Apoyo a Proyectos de Investigación e Innovación Tecnológica IN305110, de la Universidad Nacional Autónoma de México (UNAM). La correspondencia en relación con este artículo debe dirigirse a Javier Aguilar, Facultad de Psicología, UNAM, México, D.F., 04510.

Dirección electrónica:jav@unam.mx 


\begin{abstract}
The objective of this study was to develop and test a structural model of drinking among high school students based on the results of a similar study with college students, and to analyze the differences between the results of both investigations. In a sample of 190 high school students were applied the following scales: motives for drinking (compliance, coping and social-improvement), negative expectations, problems, drinking friends, attending parties, sensation seeking and three consumption measures, which were developed and / or adapted in the undergraduate study. Subsequently, scales were validated by multiple regression analysis and a structural model of drinking was developed, which showed a good fit to the data according to accepted indexes. The resulting model showed that alcohol consumption was directly affected by the above reasons, negative expectations and assistance to parties, and indirectly by sensation seeking and drinking friends. The most notable differences between high school and undergraduate are the greatest resistance to consumer pressure group in high school and greater awareness of the risks of drinking among college students.
\end{abstract}

Keywords: structural model, alcohol consumption, high school students, drinking motives, problems.

El consumo de alcohol representa un problema importante de salud pública en México. Las cifras de la Encuesta Nacional de Adicciones (2008) indican que $9.3 \%$ de la población de 12 a 65 años son bebedores consuetudinarios y 9.7 $\%$ son dependientes del alcohol. En el reporte se destaca que los adolescentes están adoptando los modelos de consumo de los adultos y una proporción importante presenta problemas con su manera de beber. Sobresalen también, el aumento del consumo de alcohol entre mujeres adolescentes y la edad cada vez más temprana de inicio del consumo.

Las variables sociales (medios, padres y compañeros) se ubican entre los factores más relevantes del consumo de drogas (Hawkins, Catalano, \& Miller, 1992; Jacob \& Leonard, 1994). Una investigación transversal entre adolescentes demostró que la mayor exposición a anuncios sobre bebidas alcohólicas se asoció a creencias más favorables acerca de los efectos del alcohol (Grube \& Wallack, 1994). Asimismo, algunos estudios han mostrado el modelamiento parental en el consumo de alcohol en adolescentes (Ennett \& Bauman, 1991); y que los niños de padres que abusan del alcohol tienen expectativas más favorables hacia su consumo (Sher, Walitzer, Wood, \& Brent, 1991).

De las variables relacionadas con el consumo de sustancias en adolescentes, el consumo por parte de los pares es consistentemente el predictor más fuerte (Newcomb \& Bentler, 1986); de su estudio y de otros se concluyó que el consumo de amigos es el determinante cercano clave del consumo del adolescente (Oetting \& Beauvais, 1986). Varios estudios han mostrado una asociación positiva entre las normas paternas y el consumo de alcohol por los adolescentes. Así Jackson, Henriksen y Dickinson (1999) 
informaron que los padres que permiten a sus hijos beber alcohol en el hogar tienen mayores probabilidades de consumir alcohol dos años después.

Un estudio transversal realizado por Van der Vorst, Engels, Meeus, Dekovic y Van Leeuwe (2005) reveló una fuerte asociación negativa entre establecer reglas parentales estrictas sobre el consumo de alcohol y la conducta de beber de adolescentes. Las consecuencias esperadas, favorables y desfavorables del consumo de bebidas alcohólicas se desarrollan desde la niñez, antes de que los niños empiecen a beber e influyen tanto en la edad de iniciación como en la frecuencia e intensidad del consumo años después.

La relación entre expectativas y la conducta de beber ha sido corroborada en diversas muestras de adolescentes (Christiansen, Goldman, \& Inn, 1982; Christiansen, Goldman, \& Brown, 1985). Se sabe que las expectativas median la relación entre los factores socioambientales, como los medios, los padres y los amigos bebedores, y la conducta de beber de los adolescentes (Hawkins, Catalano, \& Miller, 1992; Jacob \& Leonard, 1994). Por otro lado, los efectos inmediatos del consumo de alcohol, la incrementada sociabilidad y relajación, así como pleitos, daños a propiedades o agresión sexual, pueden predecir problemas de consumo y consecuencias más serias, como ser arrestado. Además, tales conductas pueden utilizarse para examinar los efectos de las intervenciones sobre las conductas problemáticas que ocurren durante los episodios de consumo (Westmaas, Moeller, \& Butler, 2007).

En México, Mora-Ríos y Natera (2001) estudiaron las relaciones entre las diferentes expectativas medidas por el Cuestionario de Expectativas del Alcohol y el Consumo entre estudiantes de universidades públicas y privadas. Entre sus hallazgos se indica que los estudiantes cuyo consumo es alto obtuvieron puntuaciones más elevadas en las subescalas de expectativas que los moderados.

Un factor clave de riesgo de consumo, tanto para las mujeres como para los hombres, es la tendencia a la rebeldía, la toma de riesgos y la búsqueda de sensaciones.

En una investigación sobre el papel de las percepciones de riesgo y personalidad entre estudiantes de bachillerato, Hampson, Severson, Burns, Slovic y Fisher (2001), encontraron que la búsqueda de sensaciones, el control del ego, el aprovechamiento escolar y la independencia se asociaron indirectamente con el consumo de alcohol. La búsqueda de sensaciones ha resultado ser un buen predictor de la participación en muchas conductas de riesgo, incluyendo el consumo de alcohol y el uso de drogas ilícitas (Donohew et al., 1999). La relación entre el primer factor y el consumo de alcohol, se ha atribuido a que los buscadores de sensaciones tienen una motivación elevada para obtener estimulación del consumo de alcohol (Read, Wood, Kahler, Maddock, \& Palfai, 2003). Otros sostienen que quienes tienen niveles altos de búsqueda de sensaciones, tienden a subestimar 
los riesgos asociados al consumo del alcohol en comparación de sus contrapartes con bajos niveles de búsqueda de sensaciones.

Dichas explicaciones se basan en un supuesto: Las tendencias a la búsqueda de sensaciones influyen de forma directa en el consumo de alcohol. Otros estudios han intentado explorar trayectorias indirectas o mediadas de los efectos de tal búsqueda sobre el consumo de alcohol; Oetting y Donnermeyer (1998) suponen que las características personales y los rasgos de personalidad, incluyendo la búsqueda de sensaciones, afectan el consumo de drogas y alcohol entre adolescentes y jóvenes sólo de modo indirecto a través de su asociación con pares consumidores de alcohol y drogas. Yanovitzky (2006) supuso que el efecto de la búsqueda de sensaciones sobre consumo de alcohol por parte de estudiantes es mediado por la presión abierta y encubierta de compañeros. Los resultados de un análisis de regresión jerárquica mostraron que la búsqueda de sensaciones influyó en el consumo de alcohol tanto directa como indirectamente, a través de su impacto sobre la frecuencia de asociación de los estudiantes con compañeros que usan alcohol.

Desde el punto de vista conceptual, los motivos para beber representan el último eslabón de una cadena mediante la cual las influencias más lejanas, como las características de personalidad, son mediadas. Los motivos han sido definidos de manera amplia como las razones conductuales conscientes o inconscientes que dirigen las energías de una persona hacia una meta (Cox \& Klinger, 1988).
La investigación de los patrones motivacionales del consumo de alcohol por parte de los adolescentes se ha asociado comúnmente con una diversidad de motivos, así, se ha encontrado que los bebedores cuyo consumo explican por razones sociales son menos propensos a presentar problemas relacionados con el consumo, comparados con quienes beben por motivos más personales (Riley, Marden, \& Lifshitz, 1948).

Cooper (1994) desarrolló y validó un modelo de cuatro factores de consumo de alcohol entre adolescentes norteamericanos de 13 a 19 años, con base en estudios de Cox y Klinger (1988, 1990). El modelo distinguió cuatro clases de motivos para beber: a) motivos de reforzamiento positivo generados internamente o motivos de mejoramiento (ejemplo, beber por placer); b) motivos de reforzamiento positivo generados externamente o motivos sociales (ejemplo, disfrutar más las reuniones sociales); c) motivos de reforzamiento negativo generados internamente o motivos de afrontamiento (ejemplo, aliviar preocupaciones y problemas personales); y d) motivos de reforzamiento negativo generados externamente o motivos de conformidad (ejemplo, no sentirse apartado del grupo).

Kuntsche, Knibbe, Gmel y Engels (2006) corroboraron las cuatro dimensiones del cuestionario de Cooper (1994) en una muestra representativa de adolescentes suizos mediante un modelo factorial confirmatorio. Asimismo, analizaron las relaciones entre las consecuencias esperadas, el consumo de alcohol y problemas asociados al alcohol, con varios modelos de 
ecuaciones estructurales. La revisión anterior proporciona evidencia de la validez de diversas variables con respecto al consumo de alcohol en adolescentes de diferentes países.

En un estudio realizado por Aguilar, Aguilar y Valencia (2011) se desarrolló y probó un modelo estructural del consumo de bebidas alcohólicas entre estudiantes de licenciatura, donde se integraron los motivos para beber de Cooper (1994), expectativas negativas y problemas derivados del consumo, así como dos factores ambientales: amigos bebedores y fiestas, y una variable de personalidad: búsqueda de sensaciones. El modelo tuvo un buen ajuste a los datos y las relaciones esperadas entre las variables consideradas resultaron significativas.

El objetivo del presente estudio fue extender la aplicación de los resultados anteriores a estudiantes de bachillerato y analizar las posibles diferencias asociadas a la menor edad de los participantes.

Para el desarrollo del modelo se dio por supuesto que el consumo sería impactado de forma positiva por la asistencia a fiestas, el afrontamiento y el mejoramiento social, y negativamente, por las expectativas negativas y la conformidad. Asimismo, se esperó que la búsqueda de sensaciones afectara positivamente asistencia a fiestas, el mejoramiento social y la convivencia con amigos bebedores.

\section{Método}

\section{Participantes}

El cuestionario se aplicó a 190 estudiantes de preparatoria pública en el Distrito Federal en sus salones de clase, a quienes se les garantizó la confidencialidad y el anonimato. Después de eliminar a los no bebedores y a los que omitieron respuestas en varios reactivos, se obtuvieron 101 cuestionarios válidos, con un rango de edad de 15 a 19 años y con una media de 16 años, de los cuales $34 \%$ fueron hombres y $66 \%$ mujeres.

\section{Instrumentos}

1. Escala de motivos de consumo de alcohol. Se tradujeron al español los reactivos de la escala de Cooper (1994) y se reemplazaron tres de ellos que no funcionaron adecuadamente en un piloteo previo. Las opciones de respuesta se redujeron de seis a cuatro, desde "definitivamente no" (1) hasta "definitivamente sí" (4).

Se realizó un análisis de componentes principales con rotación ortogonal; se obtuvieron tres factores que explican 62.47 $\%$ de la varianza. El primer factor agrupó los ocho reactivos de mejoramiento-social con cargas mayores a .50. El segundo factor agrupó siete reactivos pertenecientes a afrontamiento con cargas mayores a .60. El tercer y último factor agrupó a cuatro reactivos de conformidad con cargas mayores a 60 .

a) Mejoramiento-Social. Consta de ocho afirmaciones sobre los efectos positivos del alcohol en el estado de ánimo y en las reuniones sociales. Ejemplos: "el alcohol hace las reuniones sociales más divertidas", "me gusta cómo me siento cuando tomo alcohol". El coeficiente de confiabilidad alfa fue .88 , la media fue 18.68 y el rango de 8 a 32. 
b) Afrontamiento. Está integrado por siete reactivos referentes al manejo de emociones o pensamientos desagradables o conflictivos, mediante el consumo de alcohol. Ejemplos: "tomo para olvidar mis preocupaciones", "tomo para armarme de valor para hacer algunas cosas". El coeficiente alfa fue .89, la media fue 10.40 y el rango de 7 a 26 .

c) Conformidad. Está formada por cuatro reactivos referentes a la necesidad de ser aceptado por otros, mediante el consumo de alcohol. Ejemplos: "tomo alcohol para evitar que me molesten por no beber", "tomo alcohol para ser aceptado por el grupo de cuates". El coeficiente alfa fue .74, la media fue 5.25 y el rango de 4 a 15 .

2. Expectativas negativas. Consta de ocho reactivos que se refieren a la probabilidad de que a uno le sucedan cosas negativas al consumir alcohol. Ejemplos: "comportarme de manera ruda o grosera", "tener dificultades con mis papás”. El coeficiente alfa fue .85 , la media fue de 15.58 y el rango de 8 a 31 .

3. Problemas. Contiene nueve afirmaciones, de las cuales tres se tomaron del cuestionario de problemas de alcohol de Kuntsche et al. (2006), referentes a daños físicos o problemas con otras personas a causa del consumo de alcohol. Ejemplos: "pelearme a golpes", "problemas con mi familia". El coeficiente alfa fue .67 , la media fue de 10.85 y el rango de 9 a 21.

4. Amigos bebedores. Consta de dos aseveraciones sobre el consumo de alcohol de sus amigos y amigas. Las opciones de respuesta van de 1 (ninguno toma/nunca) a 4 (más de la mitad toma/siempre). El coeficiente alfa fue de .88 , la media fue de 8.97 y el rango fue de 4 a 12 .

5. Fiestas. Contiene dos afirmaciones referentes a la frecuencia con que asiste a bares, antros o fiestas. Las opciones de respuesta van de "nunca" (1) a "al menos una vez a la semana" (4). Los reactivos son: "yo voy a antros o bares", "yo voy a reventones, tocadas, fiestas". El coeficiente alfa fue .71, la media fue de 4.69 y el rango de 2 a 8.

6. Búsqueda de sensaciones. Contiene 15 reactivos tomados del cuestionario de Earleywine y Finn (1991), quienes se refieren al gusto por realizar cosas nuevas, impredecibles o no planeadas. Las opciones de respuesta son "verdadero" (1) y "falso" (0). Ejemplos de reactivos: "me gusta tener experiencias nuevas y excitantes", "aunque me pierda, me gusta explorar lugares que no conozco". El coeficiente alfa fue de .72, la media de 21.99 y el rango de 16 a 28.

7. Medidas de consumo. Se usaron los tres reactivos propuestos por Kuntsche et al. (2006) modificados:

1) "Durante los pasados treinta días (un mes), tomé alcohol:". Cuyas opciones de respuesta son: “0”, “1-2”, “3-5”, “6-9”, “10-19”, “2039" y "cada día, los 30 días".

2) "Durante los pasados 30 días (un mes), los días que tomé alcohol, por lo general tomé:”. Las opciones de respuesta van desde "cero bebidas", "menos de una bebida", "1", "2", “3”, "4” y "5 o más". 
3) “En los últimos 30 días, ¿cuántas veces tomaste 5 o más bebidas seguidas (una tras otra)?". Las opciones de respuesta van desde "ninguna vez", "1 vez", "2 veces", "3 a 4 veces", "5 a 6 veces", "7 a 9 veces" y "10 o más veces". En los reactivos 1 y 3 se codificaron las respuestas usando los puntos medios de las categorías, con excepción de las categorías sin rangos, las cuales se codificaron con el número asignado a cada una. En el reactivo 2, se codificaron las respuestas con los valores de 0 a 6 .

\section{Procedimiento}

El cuestionario se aplicó a 190 estudiantes de preparatoria pública en el Distrito Federal en sus salones de clase, a quienes se les garantizó la confidencialidad y el anonimato. Después de eliminar a los no bebedores y a los que omitieron respuestas en varios reactivos, se obtuvieron 101 cuestionarios válidos, con un rango de edad de 15 a 19 años y con una media de 16 años, de los cuales $34 \%$ fueron hombres y $66 \%$ mujeres.

\section{Análisis de datos}

Se empleó la prueba $t$ para grupos independientes para comparar las medias de las puntuaciones obtenidas en las diferentes escalas por los estudiantes de bachillerato y licenciatura. Se calculó el coeficiente de correlación de Pearson para cada par de escalas, así como el análisis de regresión múltiple para las tres medidas de consumo. Finalmente, se elaboró un modelo de ecuaciones estructurales para explicar el consumo promedio de bebidas alcohólicas.

\section{Resultados}

Las escalas obtenidas presentaron distribuciones ligeramente desviadas de la normalidad, tal como sucedió en el estudio con estudiantes de licenciatura.

Al comparar las medias de las puntuaciones obtenidas en las diferentes escalas por los estudiantes de bachillerato y licenciatura con la prueba $t$, se encontraron diferencias significativas en las escalas, especificadas en la tabla 1 . No se encontraron diferencias significativas entre hombres y mujeres en ninguna de las medidas de consumo.

En el bachillerato, las tres medidas de consumo tuvieron correlaciones significativas con la mayoría de las variables (ver tabla 2), pero el promedio de consumo presentó las

Tabla 1

Medias y pruebas t de escalas con diferencias significativas

\begin{tabular}{|c|c|c|c|}
\hline Escala & $\begin{array}{c}\text { Media } \\
\text { Preparatoria }\end{array}$ & $\begin{array}{c}\text { Media } \\
\text { Licenciatura }\end{array}$ & Prueba $t$ \\
\hline Amigos bebedores & 8.9 & 6.9 & $9.13, p \leq .001$ \\
\hline $\begin{array}{c}\text { Búsqueda de } \\
\text { sensaciones }\end{array}$ & 21.98 & 19.31 & $6.94, p \leq .001$ \\
\hline $\begin{array}{l}\text { Número de días de } \\
\text { consumo en el mes }\end{array}$ & 1.67 & 4.07 & $7.74, p \leq .001$ \\
\hline
\end{tabular}


correlaciones más altas, razón por la que dicha variable fue seleccionada para elaborar el modelo estructural.

El análisis de regresión múltiple del promedio de bebidas consumidas en el mes explicó el $33.60 \%$ de la varianza total, $R=.580$; cinco de las ocho escalas presentaron coeficientes de regresión parcial significativos: mejoramientosocial (Beta $=.259$ y $p \leq .015)$, afrontamiento $($ Beta $=.397$ y $p \leq .000)$, conformidad (Beta $=$ -.268 y $p \leq .003$ ), expectativas negativas (Beta $=-.174$ y $p \leq .042)$ y fiestas (Beta $=.251$ y $p$ $\leq .005)$.

Tabla 2

\section{Tabla de correlaciones}

\begin{tabular}{|l|c|c|c|c|c|c|c|c|c|c|c|}
\hline \multicolumn{1}{|c|}{ Variable } & 1 & 2 & 3 & 4 & 5 & 6 & 7 & 8 & 9 & 10 & 11 \\
\hline $\begin{array}{l}\text { 1. Número de días } \\
\text { de consumo }\end{array}$ & 1.000 & & & & & & & & & & \\
\hline $\begin{array}{l}\text { 2. Promedio de } \\
\text { bebidas }\end{array}$ & .444 & 1.000 & & & & & & & & & \\
\hline $\begin{array}{l}\text { 3. Consumo de } 5 \\
\text { más bebidas }\end{array}$ & .563 & .568 & 1.000 & & & & & & & & \\
\hline $\begin{array}{l}\text { 4. Expectativas } \\
\text { negativas }\end{array}$ & -.123 & -.204 & -.227 & 1.000 & & & & & & & \\
\hline 5. Problemas & .305 & .151 & .225 & .437 & 1.000 & & & & & & \\
\hline 6. Fiestas & .516 & .394 & .433 & -.212 & .198 & 1.000 & & & & & \\
\hline $\begin{array}{l}\text { 7. Búsqueda de } \\
\text { sensaciones }\end{array}$ & .357 & .286 & .196 & .094 & .273 & .348 & 1.000 & & & & \\
\hline 8. Amigos & .333 & .369 & .335 & -.023 & .258 & .531 & .404 & 1.000 & & & \\
\hline $\begin{array}{l}\text { 9. Mejoramiento } \\
\text { social }\end{array}$ & .415 & .512 & .469 & -.069 & .308 & .388 & .331 & .456 & 1.000 & & \\
\hline 10.Afrontamiento & .128 & .371 & .217 & .263 & .297 & .020 & .329 & .258 & .535 & 1.000 & \\
\hline 11. Conformidad & -.078 & -.055 & .039 & .236 & .202 & .011 & .139 & .135 & .259 & .465 & 1.000 \\
\hline SD & .900 & 1.757 & 1.288 & 5.503 & 2.372 & 1.433 & 3.074 & 1.972 & 6.044 & 4.409 & 1.978 \\
\hline
\end{tabular}

En los análisis de regresión múltiple de las otras dos medidas de consumo, únicamente dos escalas, fiestas y mejoramiento-social, obtuvieron coeficientes de regresión significativos.

El modelo de ecuaciones estructurales elaborado (figura 1) para explicar el consumo promedio de bebidas alcohólicas tuvo un ajuste satisfactorio a los datos, de acuerdo con los siguientes índices: $\mathrm{ji}$ cuadrada $=10.516$, g.l.$=16$ у $p \leq .838 ; N F I=.962, R F I=.914, C F I=1.000$ y $R M S A=.000$. Todos los efectos directos entre las escalas fueron significativamente diferentes de cero. Las variables con proporciones 


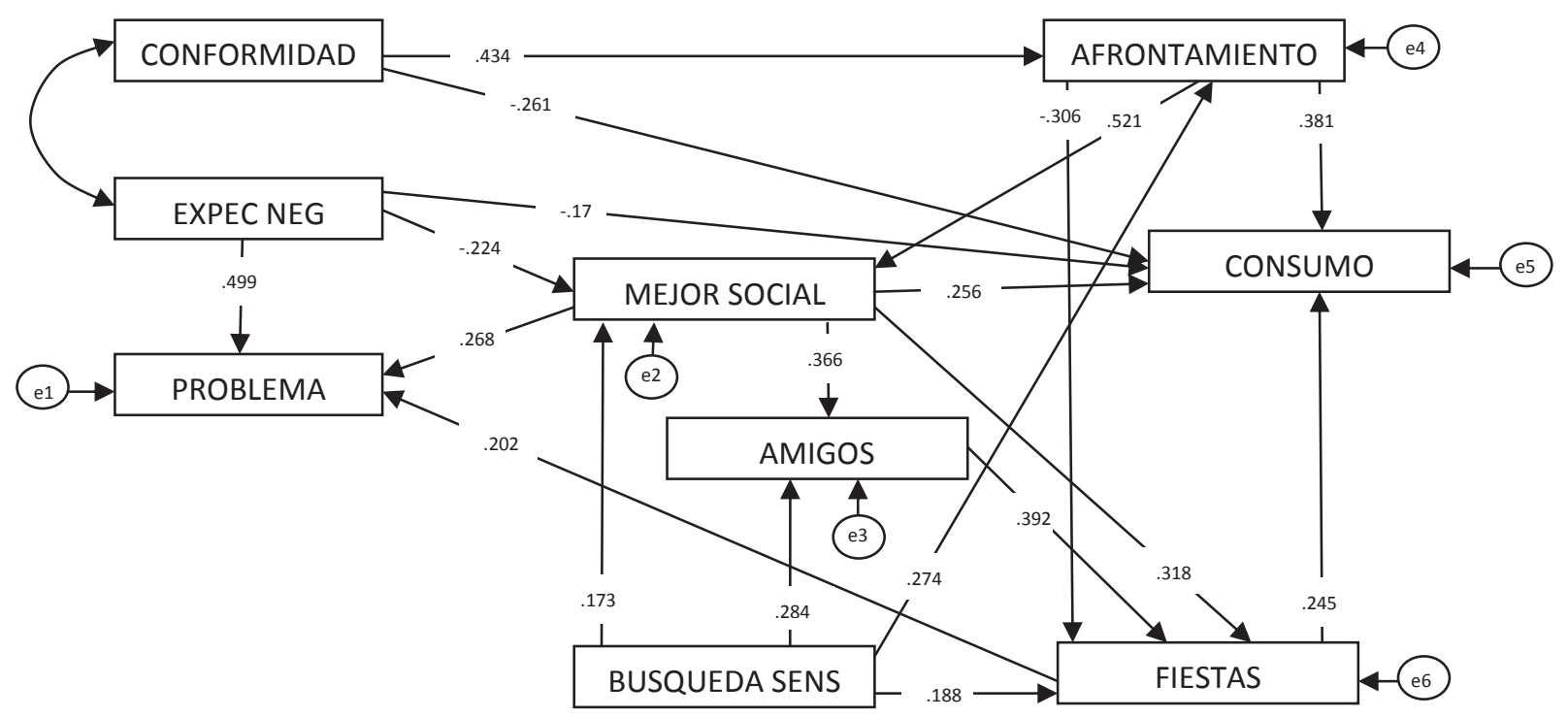

Nota. CONSUMO = consumo promedio de bebidas en el último mes; EXPEC NEG = expectativas negativas; MESOR SOCIAL = mejoramiento social; BÚSQUEDA SENS = búsqueda de sensaciones; AMIGOS = amigos bebedores.

Figura 1. Modelo estructural de consumo de promedio de bebidas.

de varianza explicada más grandes fueron: consumo promedio de alcohol, .457, fiestas, .390, y mejoramiento-social, 378 .

\section{Discusión}

En el modelo hay evidencia a favor de la hipótesis de Oetting y Donnermeyer (1998), según la cual la búsqueda de sensaciones como otros rasgos de personalidad, afectan el consumo de alcohol entre adolescentes y jóvenes sólo indirectamente a través amigos consumidores. En nuestro caso, la influencia de la búsqueda de sensaciones sobre consumo está mediada por amigos bebedores y la frecuencia de asistencia a fiestas.
El modelo obtenido es muy semejante al de los estudiantes de licenciatura, tanto por sus índices de ajuste como por las relaciones que tienen unas variables con otras. Las creencias y patrones de consumo del alcohol -característicos de un amplio sector de los adolescentes-, revisados brevemente en la introducción, explican la semejanza de los modelos estructurales de ambos niveles escolares, aunque se advierten algunas diferencias.

La relación negativa de la conformidad con el consumo entre los estudiantes de bachillerato y su ausencia entre los de licenciatura, indica que la mayor resistencia al consumo por presión de grupo en los bachilleres, evidencia una tendencia al menor consumo de bebidas alcohólicas. 
Además, entre los universitarios, el consumo se relaciona positivamente con los problemas; no así en la preparatoria, lo cual se puede explicar por la mayor conciencia de los riesgos de consumo en los primeros.

Por último, el reporte de mayores días de consumo en la universidad con respecto al de la preparatoria, es consistente con los resultados del estudio de Jaimes (2000), los cuales revelan un incremento en el consumo de alcohol a edades mayores entre estudiantes del Distrito Federal.

A pesar del cierto apoyo teórico que tienen las diferencias señaladas entre los modelos de preparatoria y licenciatura, éstas no dejan de ser hipotéticas, pues las muestras utilizadas no son representativas de las poblaciones consideradas.

\section{Referencias}

Aguilar, J., Aguilar, A., \& Valencia, A. (2011). Un modelo explicativo del consumo de alcohol entre estudiantes de licenciatura. Acta de Investigación Psicológica, 1, 491-502.

Christiansen, B. A., Goldman, M. S., \& Inn, A. (1982). Development of alcohol-related expectancies in adolescents: Separating pharmacological from social-learning influences. Journal of Consulting Clinical Psychology, 50, 336-344.

Christiansen, B. A., Goldman, M. S., \& Brown, S. A. (1985). The differential development of adolescent alcohol expectancies may predict adult alcoholism. Addictive Behaviors, 10, 299-306.
Cooper, M. L. (1994). Motivations for alcohol use among adolescents: Development and validation of a four-factor-model. Psychological Assessment, 6, 117-128.

Cox, W. M., \& Klinger, E. (1988). A motivational model of alcohol use. Journal of Abnormal Child Psychology, 97, 168-180.

Cox, W. M., \& Klinger, E. (1990). Incentive motivation, affective change, and alcohol use: A model. En W. M. Cox (Ed.), Why people drink. Parameters of alcohol as a reinforcer. (pp. 291-314). New York: Gardner Press.

Donohew, L., Hoyle, R. H., Clayton, R. R., Skinner, W. F., Colon, S. E., \& Rice, R. E. (1999). Sensation seeking and drug use by adolescents and their friends: Models for marijuana and alcohol. Journal of Studies on Alcohol, 60(5), 622-631.

Earleywine, M., \& Finn, P. R. (1991). Sensation seeking explains the relation between behavioral disinhibition and alcohol consumption. Addictive Behaviors, 16, 123128.

Ennett, S. T., \& Bauman, K. E. (1991). Mediators in the Relationship Between Parental and Peer Characteristics and Beer Drinking by Early Adolescents. Journal of Applied Social Psychology, 21, 1699-1711.

Grube, J. W., \& Wallack, L. (1994). Television beer advertising and drinking knowledge, beliefs and intentions among schoolchildren. American Journal of Public Health, 84, 254259. 
Hampson, S., Severson, H., Burns, W., Slovic, P., \& Fisher, K. (2001). Risk perception, personality factors and alcohol use among adolescents. Personality and Individual Differences, 30, 167-181.

Hawkins, J. D., Catalano, R. F., \& Miller, J. Y. (1992). Risk and protective factors for alcohol and other drug problems in adolescence and early adulthood: Implications for substance abuse prevention. Psychological Bulletin, 112, 64-105.

Instituto Nacional de Salud Pública. Encuesta Nacional de Adicciones. (2008). México: Instituto Nacional de Salud Pública (INSP), Consejo Nacional contra las Adicciones (CONADIC), Instituto Nacional de Psiquiatría Ramón de la Fuente, Fundación González Río Arronte (IAP).

Jackson, C., Henriksen, L., \& Dickinson, D. (1999). Alcohol-specific socialization, parenting behaviors and alcohol use by children. Journal of Studies on Alcohol, 60, 362-367.

Jacob, T., \& Leonard, K. (1994). Family and peer influences in the development of alcohol abuse. En R. Zucker, G. Boyd \& J. Howard (Eds.), The Development of Alcohol Problems: Exploring the biopsychosocial matrix of risk (pp. 123-155). NIAAA Research Monograph no. 26, NIH Publication no. 943495. Rockville, MD: US Department of Health and Human Services.

Jaimes, C. (2000). La evolución del consumo de drogas en estudiantes del Distrito Federal.
Recuperado de http://alcoholinformate.org. $\mathrm{mx} /$ estadisticas.cfm?articulo $=3$

Kuntsche, E., Knibbe, R., Gmel, G., \& Engels, R. (2006). Replication and validation of the drinking motive questionnaire revised (DMQ-R, Cooper, 1994) among adolescents in Switzerland. European Addiction Research, 12, 161-168.

Mora-Ríos, J., \& Natera, G. (2001). Expectativas, consumo de alcohol y problemas asociados en estudiantes universitarios de la Ciudad de México. Salud Pública de México, 43(2), 89-96.

Newcomb, M. D., \& Bentler, P. M. (1986). Substance use and ethnicity: Differential impact of peer and adult models. Journal of Psychology, 120, 83-95.

Oetting, E. R., \& Beauvais, F. (1986). Peer cluster theory: Drugs and the adolescent. Journal of Counseling and Development, 65, 17-22.

Oetting, E. R., \& Donnermeyer, J. F. (1998). Primary socialization theory: The etiology of drug use and deviance. Substance Use \& Misuse, 33(4), 995-1026.

Read, J. P., Wood M. D., Kahler, C. W., Maddock, J. E., \& Palfai, T. P. (2003). Examining the role of drinking motives in college student alcohol use and problems. Psychology of Addictive Behaviors, 17(1), 13-23.

Riley, J. W., Jr., Marden, C. F., \& Lifshitz, M. (1948). The motivational pattern of drinking based on the verbal responses of a cross-section sample of users of alcoholic beverages. 
Quarterly Journal of Studies on Alcohol, 9, 353-362.

Sher, K. J., Walitzer, K. S., Wood, P. K., \& Brent, E. E. (1991). Characteristics of children of alcoholics: Putative risk factors, substance use and abuse, and psychopathology. Journal of Abnormal Psychology, 100, 427-448.

Van der Vorst, H., Engels, R. C. M. E., Meeus, W., Dekovic, M., \& Van Leeuwe, J. (2005). The role of alcohol specific socialization in adolescents' drinking behavior. Journal of Child Psychology and Psychiatry, 47(12), 1299-1306.

Westmaas, J., Moeller, S., \& Butler, P. (2007). Validation of a measure of college students intoxicated behaviors: Associations with alcohol outcome expectancies, drinking motives and personality. Journal of American College Health, 55(4), 227-237.

Yanovitzky, I. (2006). Sensation seeking and alcohol use by collegue students: Examining multiple pathways of effects. Journal of Health Communication, 11, 269-280. 